\title{
Integrated Metabolomics-DNA
} Methylation Analysis Reveals Significant Long-Term Tissue-Dependent Directional Alterations in Aminoacyl-tRNA Biosynthesis in the Left Ventricle of the Heart and Hippocampus Following Proton Irradiation

\section{OPEN ACCESS}

Edited by:

Reza M. Salek,

International Agency For Research On

Cancer (IARC), France

Reviewed by:

Michal Jan Markuszewski,

Medical University of Gdansk, Poland

Etienne Thevenot,

Commissariat à l'Energie Atomique et

aux Energies Alternatives

(CEA), France

${ }^{*}$ Correspondence:

Jacob Raber

raberj@ohsu.edu

Specialty section: This article was submitted to

Metabolomics,

a section of the journal

Frontiers in Molecular Biosciences

Received: 10 February 2019

Accepted: 16 August 2019

Published: 10 September 2019

Citation:

Torres ERS, Hall R, Bobe G, Choi J,

Impey S, Pelz C, Lindner JR,

Stevens JF and Raber J (2019)

Integrated Metabolomics-DNA

Methylation Analysis Reveals

Significant Long-Term

Tissue-Dependent Directional

Alterations in Aminoacyl-tRNA

Biosynthesis in the Left Ventricle of the Heart and Hippocampus Following

Proton Irradiation.

Front. Mol. Biosci. 6:77.

doi: 10.3389/fmolb.2019.00077
Eileen Ruth S. Torres ${ }^{1}$, Reed Hall ${ }^{1}$, Gerd Bobe ${ }^{2,3}$, Jaewoo Choi ${ }^{2,4}$, Soren Impey ${ }^{5}$, Carl Pelz ${ }^{5}$, Jonathan R. Lindner ${ }^{6,7}$, Jan F. Stevens ${ }^{2,4}$ and Jacob Raber ${ }^{1,4,7,8 *}$

'Department of Behavioral Neuroscience, Oregon Health \& Science University, Portland, OR, United States, ${ }^{2}$ Linus Pauling Institute, Oregon State University, Corvallis, OR, United States, ${ }^{3}$ Department of Animal \& Rangeland Sciences, Oregon State University, Corvallis, OR, United States, ${ }^{4}$ Department of Pharmaceutical Sciences, College of Pharmacy, Oregon State University, Corvallis, OR, United States, ${ }^{5}$ Oregon Stem Cell Center and Department of Pediatrics, Oregon Health \& Science University, Portland, OR, United States, ${ }^{6}$ Oregon National Primate Research Center, Oregon Health \&

Science University, Portland, OR, United States, ${ }^{7}$ Knight Cardiovascular Institute, Oregon Health \& Science University, Portland, OR, United States, ${ }^{8}$ Division of Neuroscience ONPRC, Departments of Neurology and Radiation Medicine, Oregon Health \& Science University, Portland, OR, United States

In this study, an untargeted metabolomics approach was used to assess the effects of proton irradiation (1 Gy of $150 \mathrm{MeV}$ ) on the metabolome and DNA methylation pattern in the murine hippocampus and left ventricle of the heart 22 weeks following exposure using an integrated metabolomics-DNA methylation analysis. The integrated metabolomics-DNA methylation analysis in both tissues revealed significant alterations in aminoacyl-tRNA biosynthesis, but the direction of change was tissue-dependent. Individual and total amino acid synthesis were downregulated in the left ventricle of proton-irradiated mice but were upregulated in the hippocampus of proton-irradiated mice. Amino acid tRNA synthetase methylation was mostly downregulated in the hippocampus of proton-irradiated mice, whereas no consistent methylation pattern was observed for amino acid tRNA synthetases in the left ventricle of proton-irradiated mice. Thus, proton irradiation causes long-term changes in the left ventricle and hippocampus in part through methylation-based epigenetic modifications. Integrated analysis of metabolomics and DNA methylation is a powerful approach to obtain converging evidence of pathways significantly affected. This in turn might identify biomarkers of the radiation response, help identify therapeutic targets, and assess the efficacy of mitigators directed at those targets to minimize, or even prevent detrimental long-term effects of proton irradiation on the heart and the brain.

Keywords: proton irradiation, hippocampus, left ventricle, integrated epigenetic metabolomics analysis, radiation biomarkers, Parkinson's disease 


\section{INTRODUCTION}

Protons are a major component of galactic cosmic rays (GCR) and solar particle events (SPE) (Lloyd et al., 2018). Beyond the shielding of the earth's magnetic field, astronauts will be exposed to ionizing radiation (IR) from SPEs, consisting primarily of low to medium energy protons $(<\sim 150 \mathrm{MeV})$ with smaller components of helium and heavy nuclei, and to IR from GCR (Wilson, 2000). Therefore, proton irradiation as part of the space radiation environment of astronauts during missions may pose a significant long-term hazard (Simonsen et al., 1993; Cucinotta et al., 2013). This radiation exposure is also pertinent to cancer patients treated with protons (ICoR Units, 2007; Merchant et al., 2008; Lukens et al., 2015; Lupu-Plesu et al., 2017). In particular, the brain is sensitive to detrimental effects of proton irradiation in humans following cancer treatment (Merchant et al., 2008; Armstrong, 2012). Studies in rodent models are consistent with these human data (Rabin et al., 2002; Shukitt-Hale et al., 2004; Sanchez et al., 2010). Within the brain, the hippocampus might be especially sensitive to effects of proton irradiation (Rabinow et al., 2006; Bellone et al., 2014; Rudobeck et al., 2014; Parihar et al., 2015; Raber et al., 2015; Sokolova et al., 2015; Impey et al., 2016).

Compared to the hippocampus, only a few studies have been reported on the effects of proton radiation on the heart in humans. Radiation therapy for cancer treatment can lead to cardiotoxicity, suggesting that the heart is also predisposed to the effects of radiation. Additionally, there is little evidence of effective cardiac biomarkers for detecting cardiotoxicity, highlighting a need for more research (Conway et al., 2015). If the effects of proton irradiation on the heart are similar to those reported following gamma irradiation in cancer patients (Russell et al., 2009; Weintraub et al., 2010), relative late effects are anticipated (Halle et al., 2010; Lancellotti et al., 2013), and individuals in the age range of astronauts might be particularly susceptible to these long-term effects (Lancellotti et al., 2013).

Using an unbiased genome-wide approach, we previously reported that the effects of proton irradiation on the hippocampus and left ventricle 22 weeks following exposure involve tissue-dependent changes in DNA methylation, a major epigenetic modification that can impact gene expression (Impey et al., 2016). These data support increased understanding of the effects of radiation on DNA methylation in both rodent models and human cell lines (Miousse et al., 2017). Recently, research has emphasized the strong link between DNA methylation and metabolism, as some metabolic pathways are controlled by methylation-based epigenetic modifications (Chiacchiera et al., 2013). Furthermore, co-substrates generated by cellular metabolism are necessary for enzymatic control of epigenetic changes. Such alterations may even lead to disease such as cancer (Kaelin and McKnight, 2013). Diet-induced obesity also leads to changes in the epigenetic response to ionizing radiation (Vares et al., 2014). Both epigenetic modifications and altered metabolism may thus modulate the effects of ionizing radiation. As both metabolism and DNA methylation interact, we hypothesized that integrating the two would emphasize the most relevant pathways.
Recently, we combined genome-wide DNA methylation with untargeted metabolomics for an integrated analysis of the hippocampus of human apolipoprotein E mice in a high-fat diet (HFD)-induced insulin resistance (IR) mouse model (Johnson et al., 2017). This analysis revealed converging evidence for the involvement of specific pathways. In the current study, we used an untargeted metabolomics approach to assess the effects of proton irradiation ( $1 \mathrm{~Gy}$ of $150 \mathrm{MeV}$ ) on the metabolome in murine hippocampus and left ventricle of the heart 22 weeks following exposure. Additionally, we integrated these novel metabolomics data with our previous DNA methylation analysis to further identify biological pathways most affected by proton irradiation.

\section{MATERIALS AND METHODS}

\section{Animals and Study Design}

Six-month-old C57Bl6/J male mice $(n=9$ mice) were purchased from Jackson Laboratories, Bar Harbor Maine. The mice were shipped from the Jackson Laboratories to Brookhaven National Laboratory (BNL), Upton, Long Island, New York. After accommodating to the housing facility at BNL for 1 week, the mice were transported to the NASA Space Radiation Laboratory (NSRL) on the BNL campus and irradiated with $1 \mathrm{~Gy}$ of 150 $\mathrm{MeV}$ protons or sham-irradiated. The mice were loaded into 8 $\times 3 \times 3 \mathrm{~cm}$ plastic square enclosures. These enclosures were either placed in a foam fixture in the beam line and exposed to a rectangular beam of $\sim 20 \times 20 \mathrm{~cm}$ generated by the Booster accelerator at $\mathrm{BNL}$ and transferred to the experimental beam line at the NSRL facility or received sham irradiation for the same time as the irradiated mice ( $n=4-5$ mice/exposure condition; the mice were randomly assigned to the two groups). Dose calibration was performed to obtain the targeted dose. The week following the irradiation or sham irradiation, the mice were shipped to Oregon Health \& Science University (OHSU) and group-housed under standard care for 22 weeks after which they were euthanized by cervical dislocation for tissue analyses. The hippocampus of one hemibrain and half of the left ventricle of nine mice were divided into separate tissues for DNA methylation analyses. The hippocampus of the other hemibrain and the other half of the left ventricle were processed for untargeted metabolomics. The researchers were blinded to the exposure condition until after completion of all DNA methylation and metabolomics data acquisition and analyses. All protocols were reviewed and approved by the Institutional Animal Care and Use Committees (IACUC) of BNL and OHSU and in compliance with all federal regulations. Animal experiments were carried out in accordance with the relevant guidelines and regulations.

\section{Metabolomics}

Hippocampal and left ventricular tissues were taken from sham-irradiated and proton-irradiated mice. These tissues were dissected and homogenized in RIPA $(500 \mu \mathrm{l})$. Metabolites were extracted from $100 \mu \mathrm{l}$ of hippocampal homogenates and $100 \mu \mathrm{l}$ of left ventricular homogenates and untargeted metabolomics was completed as described (Kirkwood et al., 2013). Briefly, a Shimadzu Nexera system was used to run high-pressure liquid 
chromatography coupled to a quadrupole time-of-flight-mass spectrometer (Sciex TripleTOF 5600). This was operated in datadependent MS/MS (IDA) acquisition mode in both positive and negative ion mode (Kirkwood et al., 2012).

Metabolomics data were processed using Markerview and Peakview software (AB Sciex, Framingham, MA, U.S.A.). Metabolites were initially identified by comparisons with an in-house library consisting of 619 IROA standards (IROA Technologies, Bolton, MA, U.S.A.) and 30 other additional commercially available standards. Additional metabolites were individually identified based on MS isotopic pattern, accurate mass (mass error $<5 \mathrm{ppm}$ ), MS/MS fragment ions, and matching with spectra in online databases, including Metlin, Human Metabolome Database (HMDB), and Lipidmaps.

To assess analytical variability compared to biological variation, we included four technical replicate analyses of a quality control (QC) sample, which was prepared by mixing $5-\mu \mathrm{l}$ aliquots of all nine biological processed samples from the protonirradiated and sham-irradiated mice, and visualized the noncentered, Pareto scaled data by Principal Component Analysis of hippocampal tissue (Supplementary Figure 1A), and ventricle tissue (Supplementary Figure 1B). These data show that the variation in the technical replicates is negligible compared to the biological variation.

\section{DNA Methylation}

DNA methylation was performed as described (Impey et al., 2016, 2017). Briefly, DNA was isolated from the left ventricle and hippocampus in the same mice processed for metabolomics analyses. Antibodies against 5-methylcytosine $(5 \mathrm{mC})$ and 5 hydroxymethylcytosine $(5 \mathrm{hmC})$ that do not cross react were used to immunoprecipitate DNA fragments (DIP). Eight pools of tissues $(2 \times 2$ pools of hippocampal tissues and $2 \times 2$ pools of left ventricle tissues, or 2 pools/tissue/radiation condition) were used. For DIP-Seq library preparation, RNAse-treated DNA was isolated using the Qiagen Allprep DNA/RNA protocol, involving "randomly" fragmenting dsDNA fragmentase (NEB), polishing using the DNA terminator end repair kit (Lucigen), and adding an "A" base to the 3 ' end of DNA fragments using Klenow exo(Epicenter). The genomic DNA was ligated to un-methylated HT TrueSeq indexed adapters, purified, denatured, resuspended in $100 \mu \mathrm{l}$ of DIP IP buffer, and immunoprecipitated, and the eluted DNA was purified and subjected to limited amplification ( $\sim 15$ cycles $)$.

Illumina high-throughput genomic sequencing of the immunoprecipitated meDIP DNA (DIP-Seq) on a HiSeq2000 platform was used to identify differentially methylated regions and associated genes throughout the genome. We generated a bioinformatic pipeline to map and annotate DIP-Seq sequencing and statistical algorithms that allow for the identification of differentially methylated regions in DIP-Seq data sets.

\section{Statistical Analyses}

To identify pathways (and associated metabolites) that are affected by radiation, we used a multi-step statistical analysis approach based in part on previous analyses (Xia and Wishart, 2010; Kirkwood et al., 2013). In the first exploratory phase, we used an untargeted metabolomics approach and determined for the metabolomics data the top 5 pathways with at least 3 identified metabolites that were altered by radiation treatment. Signal intensities of identified metabolites were annotated with HMDB numbers. Annotated, non-transformed signal intensities were Pareto-scaled in MetaboAnalyst 4.0 (Chong et al., 2018). For pathway analysis using known Mus musculus KEGG pathways in MetaboAnalyst, only metabolites with HMDB number were included. For multi-comparison adjustment, false-discovery rates (FDR) are reported (Benjamini and Hochberg, 1995). The R scripts generated are included as Supplementary File $\mathbf{1}$.

For the integrated DNA methylation metabolomics analysis, we first reanalyzed the DNA methylation data of our original publication (Impey et al., 2016). Briefly, regions of methylation enrichment (i.e., $5 \mathrm{hmC}$ regions) were merged. Treatment group differences were determined by negative binomial tests. Gene ontology analyses involved the bioconductor Goseq package, which adjusts for RNA-Seq length bias artifacts. To confirm that the re-analyzed DNA methylation did not differ from those in our original publication (Impey et al., 2016), pathway data were visualized using R/Bioconductor (Roswell Park Cancer Institute, Buffalo, NY) or Cytoscape software (Shannon et al., 2003). The reanalyzed data gave similar results as our original publication (Impey et al., 2016) and are posted on the NIH Gene Expression Omnibus (GEO) web site (accession number: GSE132588). The $\mathrm{C}$ source code will be provided upon request. DNA methylation data were then merged with metabolomics data in MetaboAnalyst using only methylation regions with treatment group differences with a raw $p$-value of $<0.01$. The $\mathrm{R}$ scripts used for MetaboAnalyst are included as a .zip file as Supplementary File 2. In short, pathway analyses and enrichment analyses were set to "hypergeometric test," topology analysis set to "degree centrality," and "gene-metabolite pathways" were chosen, as described (Johnson et al., 2017). After FDR adjustment, the aminoacyl-tRNA biosynthesis pathway was identified as the only significantly altered pathway.

In the second, targeted phase, in which we show that radiation altered metabolite abundance as well as methylation of the amino-tRNA biosynthesis pathway, we focused on the 15 identified amino acids of the amino-tRNA biosynthesis pathways as well as the methylation status of their tRNA synthetases and did not adjust for multiple comparisons. Non-transformed signal intensities of the 15 amino acids of the aminoacyl-tRNA biosysnthesis pathway were Pareto-scaled in MetaboAnalyst and the effect of radiation was visually examined using partial least squares-discriminant analysis (PLS-DA) in MetaboAnalyst. The visual examination identified one mouse sample (number 11) as an outlier. Therefore, the statistical comparison between sham vs. proton-irradiated mice using the non-parametric KruskalWallis test was done once with and once without number 11 . In addition, we used an unpaired student's test to compare sham-irradiated vs. proton-irradiated methylation patterns of amino acid tRNA synthetases. All statistical tests were two-sided. Significance was set at $p \leq 0.05$ and a statistical tendency at $p<0.10$ unless otherwise noted.

Figures were generated using MetaboAnalyst 4.0 (Quebec, Canada), GraphPad Prism v6 (La Jolla, CA), and GIMP (http:// 

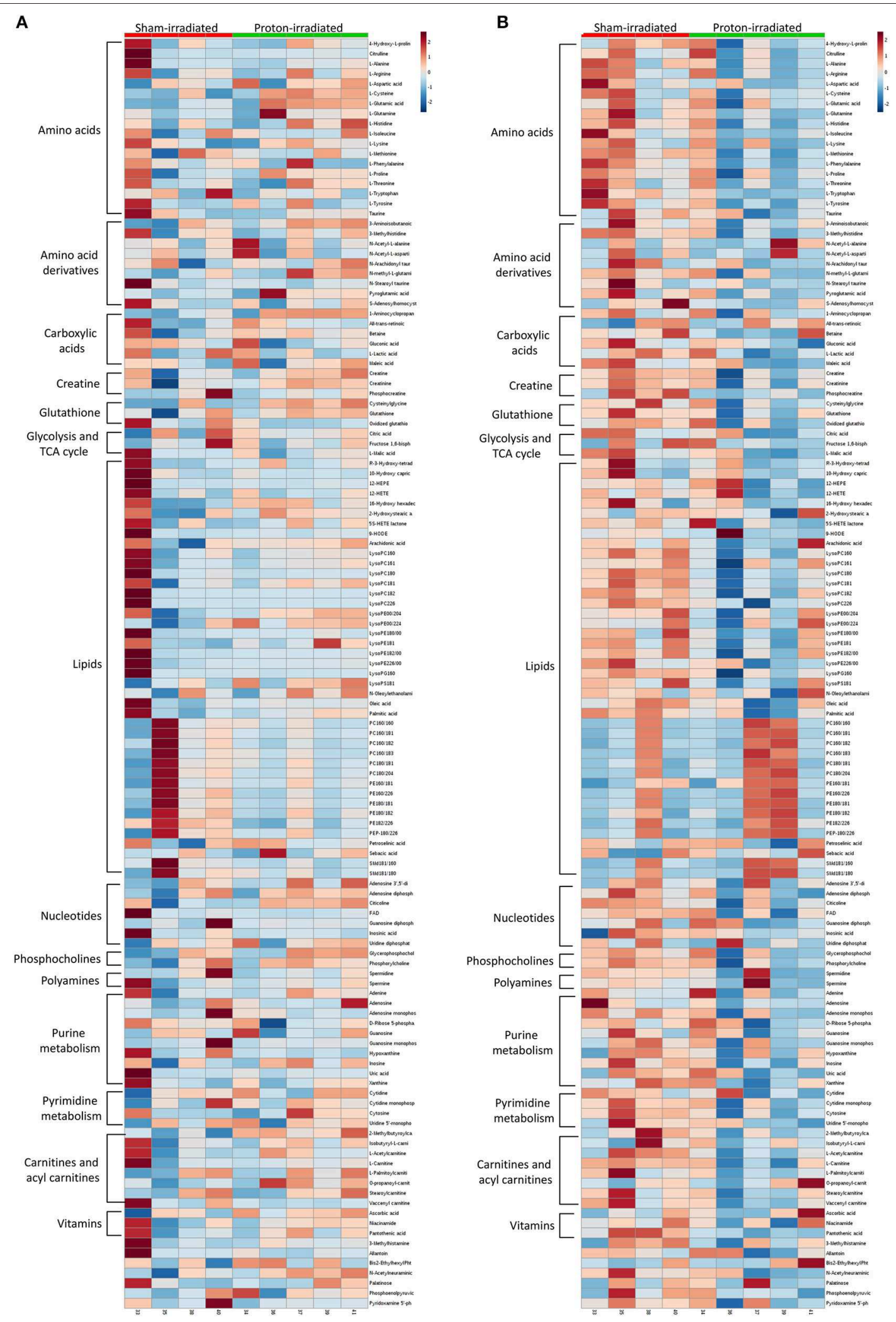

FIGURE 1 | Heatmaps of metabolomics analyses of the hippocampus (A) and left ventricle (B) 22 weeks following proton and sham irradiation. 
GIMP.org). To provide a comparable scale, changes in individual and total amino acids are shown as \% changes from shamirradiated mice (median proton-irradiated mice/median shamirradiated mice in \% -100) in Figure 3.

\section{RESULTS}

\section{Metabolite Identification}

Using untargeted metabolomics, we detected and semi-quantified mass spectral features in hippocampal, and left ventricle heart tissues of proton irradiated and sham-irradiated mice. Using accurate mass and MS/MS and isotope ratio patterns, we assigned 127 features to metabolite identities using online database searching, 78 of which were confirmed by our inhouse library of metabolite standards using PeakView software (Supplementary Tables 1, 2). There were a small proportion of metabolites that were identified in both the positive and negative ion mode. For these, we evaluated them using the student's $t$ test and chose the ion mode data for each metabolite found in duplicate based on its abundance and levels of significance.

\section{Metabolomics Analysis Hippocampus}

Of the 52 identified KEGG pathways, no pathways were significantly altered by proton irradiation in hippocampal tissue, although several pathways trended toward significance (see Supplementary Table 3 for top 5 of 15 pathways with at least 3 identified metabolites). The heat map analysis of individual metabolites is visualized in Figure 1A. The only pathway that ranked in the top 5 pathways for each tissue and each analysis (Supplementary Tables 3, 4) was the aminoacyl-tRNA biosynthesis pathway. Based on these findings, we focused our further statistical analysis on the aminoacyl-tRNA biosynthesis pathway. A PLS-DA analysis visualized that the 15 identified amino acids of the aminoacyl-tRNA biosynthesis pathway could differentiate between sham- and proton-irradiated mice (Figure 2A). The first principal component, which differentiated all but one proton-irradiated mouse (number 11), accounted for $56.2 \%$ of the variability. Next, we examined each of 15 identified amino acids individually or as a sum. Using the non-parametric Wilcoxon Rank Sum test, cysteine was $83 \%$ higher in protonthan sham-irradiated mice $(p=0.01)$, and glutamate tended to be $23 \%$ higher in proton- than sham-irradiated mice $(p=0.08)$. The sum of all 15 amino acids tended to be $12 \%$ higher in protonthan sham-irradiated mice ( $p=0.08$; Figure 3A). After exclusion of mouse number 11, mice of the two groups differed in the levels of cysteine, glutamate, and total amino acids, with higher values in the proton-irradiated mice and a $p$-value of 0.02 .

\section{Metabolomics Analysis Left Ventricle of the Heart}

Of the 52 identified KEGG pathways, no pathways were significantly altered following FDR adjustment by proton irradiation in the left ventricle of the heart (see Supplementary Table 3 for top 5 of 15 pathways with at least 3 identified metabolites). The heat map analysis of individual metabolites is visualized in Figure 1B. A PLS-DA analysis showed that the 15 identified amino acids of the aminoacyltRNA biosynthesis pathway differentiated between shamand proton-irradiated mice (Figure 2B). The first principal component, which differentiated all but one proton-irradiated mouse (number 11), accounted for $80.4 \%$ of the variability and the second another $11.6 \%$, indicating an even greater impact of proton irradiation on the left ventricle than on the hippocampus. In contrast to the hippocampus, protein irradiation consistently decreased protein values for all 15 amino acids. Significant decreases were observed for 3 of 15 amino acids, namely phenylalanine $(-41 \% ; p=0.05)$, tryptophan $(-34 \% ; p=0.05)$, and methionine $(-36 \% ; p=0.05)$, and a tendency for another 4 amino acids, namely alanine $(-32 \% ; p=0.09)$, glutamine $(-25 \% ; p=0.09)$, histidine $(-18 \% ; p=0.09)$, and tyrosine $(-50 \% ; p=0.09)$. The sum of all 15 amino acids tended to be $31 \%$ lower in proton- vs. sham-irradiated mice ( $p=0.09$; Figure 3B). As was for the hippocampus, our analysis only misclassified mouse number 11. After exclusion of number 11, mice of the two groups perfectly separated for 4 of 15 amino acids (histidine, phenylalanine, tryptophan, and methionine) with lower values for proton-than sham-irradiated mice and a $p$-value of 0.02 . One misclassification and a $p$-value of 0.04 was achieved for total amino acids and another 3 amino acids (glutamine, isoleucine, and tyrosine).

\section{Comparison Between Metabolites in the Left Ventricle of the Heart and Those in Hippocampus}

To evaluate whether proton irradiation affected the aminoacyltRNA biosynthesis pathway differently in the left ventricle and the hippocampus, we calculated the ratio of the left ventricle and hippocampus for each amino acid. Consistent with the pattern in the left ventricle, the ratio for each amino acid went down following proton irradiation. PLS-DA analysis with those 15 amino acid ratios could separate sham- and proton-irradiated mice (Figure 2C). Significant decreases were observed for 3 of 15 amino acids, namely phenylalanine, isoleucine, and tyrosine (all $p=0.05$ ), and a tendency for another 3 amino acids, namely arginine, aspartate, and histidine (all $p=0.09$; Figure $3 \mathrm{C}$ ). The ratio for the sum of all 15 amino acids was lower in proton- than sham-irradiated mice $(p=0.05)$. The only misclassified mouse was number 11. After exclusion of number 11, the mice of the two groups perfectly separated for total amino acid ratio and 3 amino acid ratios (arginine, histidine, and isoleucine) with lower values for proton-irradiated than sham-irradiated mice and a $p$-value of 0.02 . One misclassification and a $p$-value of 0.04 was achieved for another 4 amino acids (glutamate, glutamine, phenylalanine, and tyrosine).

\section{Integrated Metabolomics-DNA Methylation Analysis of the Hippocampus}

We used our updated bioinformatic pipeline to reanalyze the left ventricle and hippocampal DNA methylation reported in the original publication [(Impey et al., 2016); see GEO web site accession number: GSE132588]. Next, we integrated DNA methylation data and metabolomic data using the joint pathway 

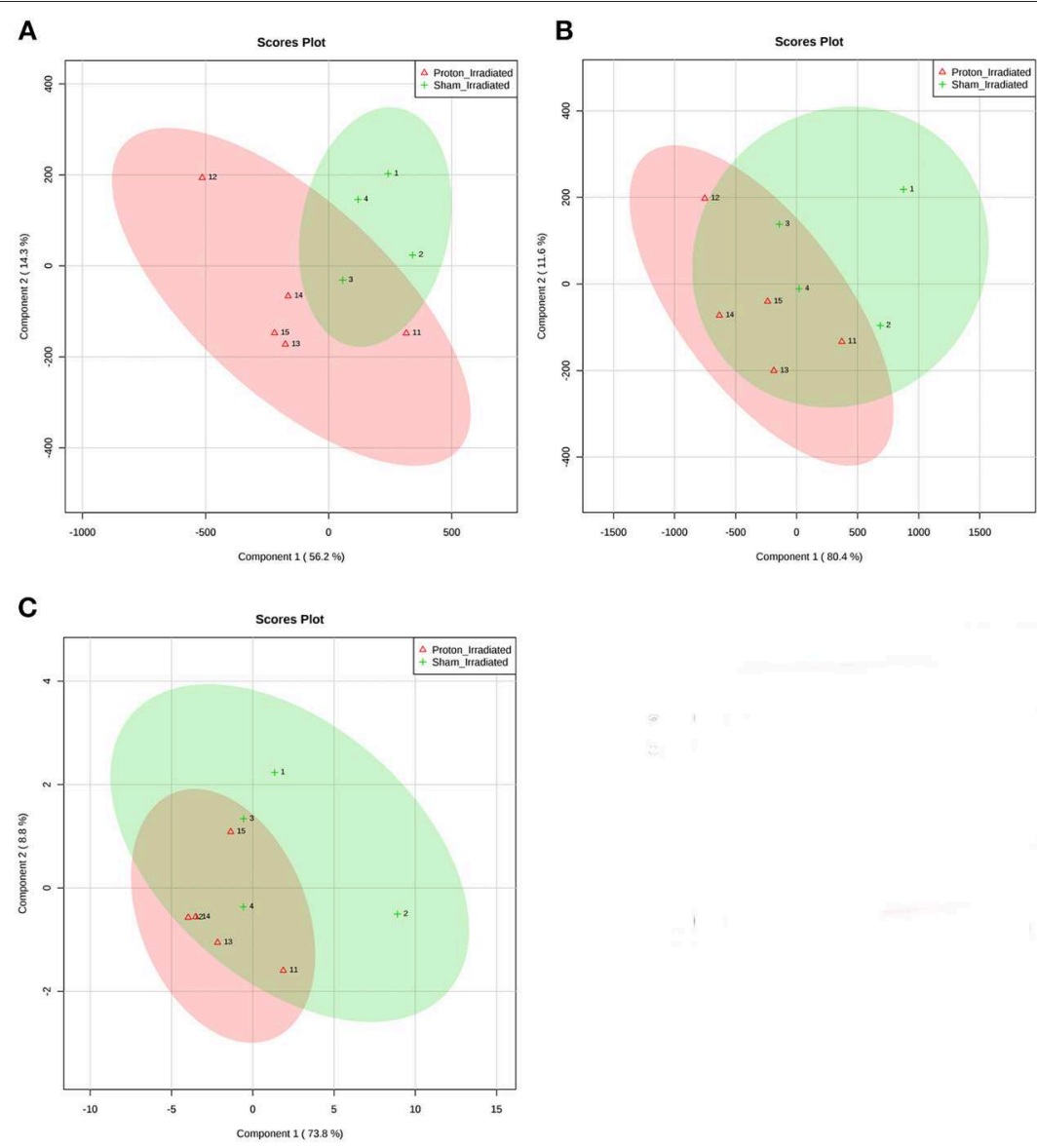

FIGURE 2 | Partial least squares discriminant analysis (PLS-DA) of (A) hippocampal tissue, (B) left ventricular tissue, and (C) the ratio of left ventricular tissue and hippocampal tissue discriminates between sham- and proton-irradiated mice. Signal intensities of 15 individual amino acids were scaled (Pareto scaling) and then analyzed using PLS-DA. The ellipses were drawn at $95 \% \mathrm{Cl}$ of normal distribution for a given group. Loading plots illustrate the proportion of variation accounted for by each component. Each number represents one mouse (1-4 for sham-irradiated mice, and 11-15 for proton-irradiated mice).

analysis function of MetaboAnalyst, which compared alterations in DNA methylation profiles and metabolic pathways using KEGG annotations as the reference frame.

The integrated analysis of hippocampal tissue revealed significant alterations following FDR adjustment in the aminoacyl-tRNA biosynthesis pathway (Supplementary Table 4). Of the 15 amino acids, we identified tRNA synthetase methylation of 9 amino acids. Proton irradiation resulted in significantly lower values in tRNA synthetase methylation of $44 \%$ ( 4 of 9) of amino acids, namely threonine $(-80 \% ; p=0.005)$, tryptophan $(-79 \% ; p=0.007)$, phenylalanine $(-75 \% ; p=0.01)$, and isoleucine $(-72 \%$; $p=0.02)$, and a tendency for arginine $(-70 \% ; p=0.07)$. The pattern of down-regulation was consistent as only 1 of 9 amino acid tRNA synthetases had higher methylation values (cysteine: $+77 \%$; $p=0.36$ ) in proton- than sham-irradiated mice (results not shown).

\section{Integrated Metabolomics-DNA Methylation Analysis of the Left Ventricle}

The integrated analysis of left ventricular tissue also revealed significant alterations in aminoacyl-tRNA biosynthesis (Supplementary Table 4). Next, we combined our metabolomics and DNA methylation results for the aminoacyl-tRNA biosynthesis pathway. Of the 15 amino acids, we identified tRNA synthetase methylation of 3 amino acids. Proton irradiation resulted in significantly higher values in tRNA synthetase methylation of phenylalanine $(+562 \% ; p=0.02)$, whereas isoleucine and threonine were not significantly affected. However, we observed significantly lower values in tRNA synthetase methylation of asparagine $(-92 \% ; p=0.002)$. No consistent methylation pattern was observed comparing proton- vs. sham-irradiated mice.

\section{DISCUSSION}

The data of the current study support the value of metabolomics and especially integrated analyses of metabolomics and DNA methylation to identify long-term changes in distinct tissues following whole body radiation. Below we first discuss the metabolomics analysis by itself, followed by the integrated metabolomics DNA methylation analysis. 


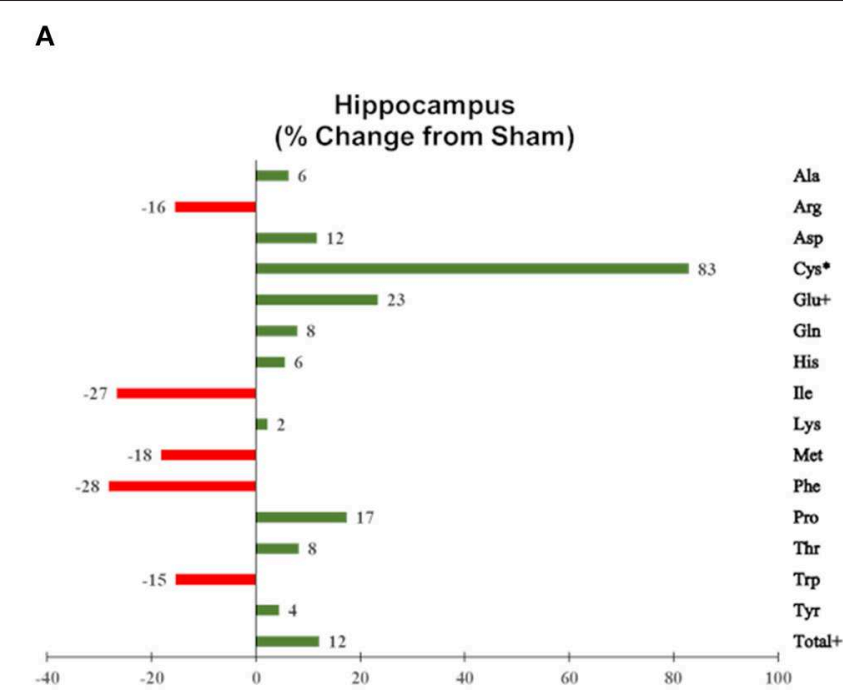

\section{B}

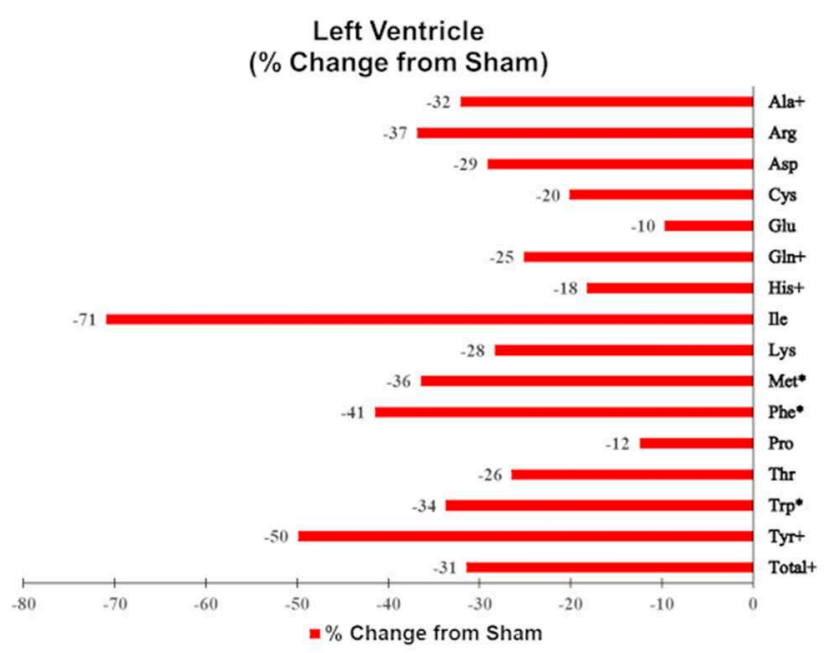

C

Ratio Left Ventricle: Hippocampus

(\% change from Sham)

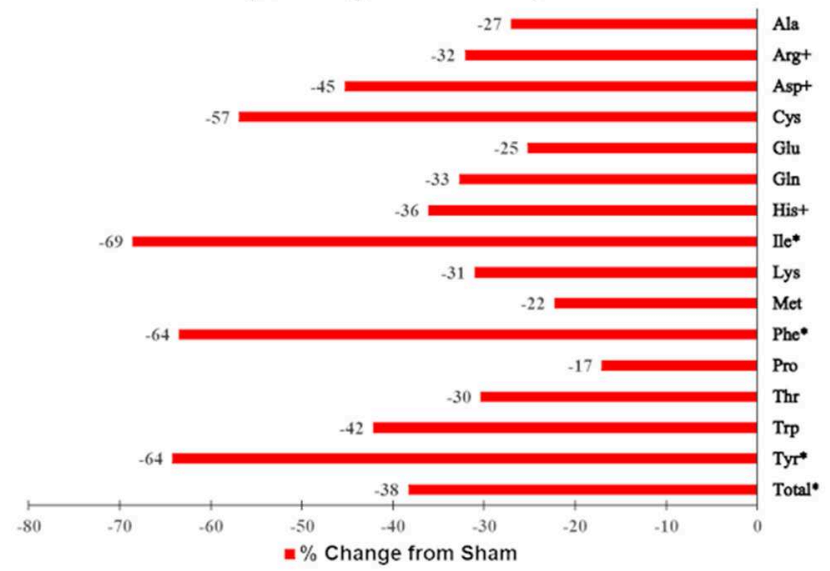

FIGURE 3 | Effects of proton irradiation on signal intensities of individual and total amino acids in (A) hippocampal tissue, (B) left ventricular tissue, and (C) the ratio of left ventricular tissue and hippocampal tissue in sham-, and proton-irradiated mice. Results are shown as \% changes from sham-irradiated mice (median proton-irradiated mice/median sham-irradiated mice in $\%-100$ ). Amino acids with a * signify a $p \leq 0.05$ and amino acids with a + signify a $0.05<p \leq 0.10$.

Using untargeted metabolomics analysis by itself, the current study was not able to identify pathways that were significantly altered by proton irradiation in the hippocampus and the left ventricle of the heart using FDR adjustment. However, the pathway analysis revealed aminoacyl-tRNA biosynthesis as the most promising pathway to further pursue for targeted analysis. Previous work in metabolic profiling of urine samples from C57BL/6J mice $4 \mathrm{~h}$ following proton irradiation (0.5 and $2 \mathrm{~Gy}$ of $1 \mathrm{GeV}$ ) revealed profound alterations in energy metabolism, amino acid metabolism, and purine and pyrimidine metabolism (Laiakis et al., 2015). Taken with our findings, this suggests that proton irradiation may cause early systemic changes in biofluids, with long-lasting changes in tissues. Thus, even a single proton irradiation event can lead to longterm changes.

Aminoacyl-tRNA biosynthesis, key for protein synthesis including the accuracy of translation (Ibba and Söll, 2004), was significantly altered (FDR adjusted) by proton irradiation in the hippocampus and left ventricle as demonstrated by the integrated metabolomics-DNA methylation analysis. Interestingly, aminoacyl-tRNA biosynthesis was recently identified as one of two metabolic pathways most strongly associated with left ventricular diastolic dysfunction, and consistent over a 5 year period, in 570 randomly recruited 
people (Zhang et al., 2013). The downregulation of metabolites in this pathway in the left ventricle of proton-irradiated mice is especially concerning as deficiency in tRNA synthetase editing activity can cause cardioproteinopathy (Liu et al., 2014). Mutations in aminoacyl-tRNA synthetases have been linked to several disease conditions, including cancer and various neurodegenerative conditions like Parkinson's Disease (Park et al., 2008; Sauter et al., 2015; Kapur et al., 2017; Meyer-Schuman and Antonellis, 2017). Hence further analysis is warranted considering its role in cardiovascular function.

In summary, proton irradiation causes long-term changes in the left ventricle and hippocampus. The aminoacyl tRNA biosynthesis pathway might be especially valuable as a biomarker of the proton radiation response. Future studies should address to what extent this pathway could be targeted to attenuate detrimental long-term effects of proton irradiation on the heart and the brain. Thus, integrated analysis of metabolomics and DNA methylation is a powerful approach to obtain converging evidence of pathways significantly affected by proton irradiation. Our findings also suggest the importance of assessing tissue-specific compensatory changes. Such tissuedependent compensatory directional changes will likely depend on the time interval after radiation exposure. A limitation of our study is that we included only behaviorally naïve mice, as behavioral testing would be expected to affect DNA methylation and metabolic pathways and we wanted to assess the effects of proton irradiation by itself. As permutation and cross-validation tests require relative large data sets and the statistical power of ranking tests is limited, we did not adjust for multiple comparisons in the targeted analysis of the 15 identified amino acids of the amino-tRNA biosynthesis pathways as well as the methylation status of their tRNA synthetases. Therefore, future studies are warranted to compare radiation responses at distinct intervals following radiation exposure and involving larger group sizes and comparisons between behaviorally naïve and behaviorally tested mice following proton irradiation.

\section{REFERENCES}

Armstrong, F. (2012). Proton-beam radiation therapy and health-related quality of life in children with CNS tumors. J. Clin. Oncol. 30, 2028-2029. doi: 10.1200/JCO.2012.42.1248

Bellone, J., Hartman, R., and Vlkolinsky, R. (2014). The effects of low doses of proton, iron, or silicon radiation on spatial learning in a mouse model of Alzheimer's disease. J. Radiat. Res. 55(Suppl. 1), i95-i96. doi: 10.1093/jrr/rrt154

Benjamini, Y., and Hochberg, Y. (1995). Controlling the false discovery rate: a practical and powerful approach to multiple testing. J. R. Stat. Soc. B 57, 289-300. doi: 10.1111/j.2517-6161.1995.tb02031.x

Chiacchiera, F., Piunti, A., and Pasini, D. (2013). Epigenetic methylations and theri connections with metabolism. Cell. Mol. Life Sci. 70, 1495-1508. doi: $10.1007 / \mathrm{s} 00018-013-1293-5$

Chong, J., Soufan, O., Li, C., Caraus, I., Li, S., Bourque, G., et al. (2018). MetaboAnalyst 4.0: towards more transparent and integrative metabolomics analysis. Nucleic Acids Res. 46, w486-w494. doi: 10.1093/nar/gky310

Conway, A., McCarthy, A. L., Lawrence, P., and Clark, R. A. (2015). The prevention, detection and management of cancer

\section{ETHICS STATEMENT}

This study was carried out in accordance with the recommendations of the OHSU and BNL IACUC committee. The protocol was approved by the OHSU and BNL IACUC committees.

\section{AUTHOR CONTRIBUTIONS}

SI, JS, and JR conceived and planned this study. ET, RH, GB, JC, $\mathrm{CP}$, and SI analyzed the data. All authors discussed the results and contributed to the final manuscript.

\section{FUNDING}

This work was supported by NASA grants NNJ12ZSA001N and NSCOR NNX15AK13G, and National Institutes of Health grant S10RR027878.

\section{ACKNOWLEDGMENTS}

The authors wish to thank Charity Miltenberger, Sky Nelson, and Derek Wong for their help identifying the metabolites and Peter Guida, Adam Rusek, and other NSRL physics staff for their invaluable help at BNL. Additionally, we thank Jeff Morré at OSU for assisting with the metabolomics analyses. We also thank Dr. Searles, the Director of the Massively Parallel Sequencing Shared Resource and Associate Director Integrated Genomics Laboratory at OHSU, for providing his expertise for the design and interpretation of the DNA methylation experiments in this study as well as Dr. Jessica Minnier for her advice regarding the statistical analyses.

\section{SUPPLEMENTARY MATERIAL}

The Supplementary Material for this article can be found online at: https://www.frontiersin.org/articles/10.3389/fmolb. 2019.00077/full\#supplementary-material

treatment-induced cardiotoxicity: a meta-review. BMC Cancer 15:366. doi: 10.1186/s12885-015-1407-6

Cucinotta, F. A., Kim, M. H., Chappell, L. J., and Huff, J. L. (2013). How safe is safe enough? radiation risk for a human mission to Mars. PLoS ONE 8:e74988. doi: 10.1371/journal.pone.0074988

Halle, M., Gabrielsen, A., Paulsson-Benne, G., Gahm, C., Agardh, H. E., and Farnebo, F. (2010). Sustained inflammation due to nuclear factor-kappa B activation in irradiated human arteries. J. Am. Coll. Cardiol. 55, 1227-1236. doi: 10.1016/j.jacc.2009.10.047

Ibba, M., and Söll, D. (2004). Aminoacyl-tRNAs: setting the limits of the genetic code. Genes Dev. 18, 731-738. doi: 10.1101/gad.1187404

ICoR Units (2007). Prescribing, recording, and reporting proton-beam therapy: contents. J. Int. Comm. Radiation Units Measur. 7. doi: 10.1093/jicru/ndm021

Impey, S., Jopson, T., Pelz, C., Tafessu, A., Fareh, F., Zuloaga, D., et al. (2017). Bi-directional and shared epigenomic signatures following proton and 56Fe irradiation. Sci. Rep. 7:10227. doi: 10.1038/s41598-017-09 191-4

Impey, S., Pelz, C., Tafessu, A., Marzulla, T., Turker, M. S., and Raber, J. (2016). Proton irradiation induces persistent and tissue-specific DNA methylation 
changes in the left ventricle and hippocampus. BMC Genomics 17:273. doi: 10.1186/s12864-016-2581-x

Johnson, L. A. E., Torres, R. S., Impey, S., Stevens, J. F., and Raber, J. A. (2017) polipoprotein $\mathrm{E} 4$ and insulin resistance interact to impair cognition and alter the epigenome and metabolome. Sci. Rep. 7:43701. doi: 10.1038/srep43701

Kaelin, W. G. Jr., and McKnight, S. L. (2013). Influence of metabolism on epigenetics and disease. Cell 153, 56-69. doi: 10.1016/j.cell.2013.03.004

Kapur, M., Monaghan, C. E., and Ackerman, S. L. (2017). Regulation of mRNA translation in neurons-a matter of life and death. Neuron 96, 616-637. doi: 10.1016/j.neuron.2017.09.057

Kirkwood, J. S., Lebold, K. M., Miranda, C. L., Wright, C. L., Miller, G. W., Tanguay, R. L., et al. (2012). Vitamin C deficiency activates the purine nucleotide cycle in zebrafish. J. Biol. Chem. 287, 3833-3841. doi: $10.1074 /$ jbc.M111.316018

Kirkwood, J. S., Maier, C. S., and Stevens, J. F. (2013). Simultaneous, untargeted metabolic profiling of polar and non-polar metabolites by LC-Q-TOF mass spectrometry. Curr. Protoc. Toxicol. 4:4.39. doi: 10.1002/0471140856.tx0439s56

Laiakis, E., Trani, D., Moon, B.-H., Strawn, S., and Fornace, A. J. Jr. (2015). Metabolic profiling of urine samples from mice exposed to protons reveals radiation quality and dose specific differences. Radiat. Res. 183, 382-390. doi: $10.1667 /$ RR 3967.1

Lancellotti, P., Nkomo, V. T., Badano, L. P., Bergler-Klein, J., Bogaert, J., Davin, L., et al. (2013). Expert consensus for multi-modality imaging evaluation of cardiovascular complications of radiotherapy in adults: a report from the European Association of cardiovascular imaging and the American society of echocardiography. J. Am. Soc. Echocardiogr. 26, 1013-1032. doi: 10.1016/j.echo.2013.07.005

Liu, Y., Satz, J. S., Vo, M.-N., Nangle, L. A., Schimmel, P., and Ackerman, S. L. (2014). Deficiencies in tRNA synthetase editing activity cause cardioproteinopathy. Proc. Natl. Acad. Sci. U.S.A. 111, 17570-17575. doi: 10.1073/pnas.1420196111

Lloyd, C., Townsend, S., Reeves, K., et al. (2018). NASA Human Research Program Engagement and Communiciations. Radiation iBook. Available online at: https://www.nasa.gov/sites/default/files/atoms/files/nasa_space_radiation_ ebook_0.pdf (accessed October 30, 2017).

Lukens, J. N., Lin, A., and Hahn, S. M. (2015). Proton therapy for head and neck cancer. Curr. Opin. Oncol. 27, 165-171. doi: 10.1097/CCO.0000000000000181

Lupu-Plesu, M., Claren, A., Martial, S., N’Diaye, P.-D., Lebrigand, K., Pons, N., et al. (2017). Effects of proton versus photon irradiation on (lymph)angiogenic, inflammatory, proliferative and anti-tumor immune responses in head and neck squamous cell carcinoma. Oncogenesis 6:e354. doi: 10.1038/oncsis.2017.56

Merchant, T. E., Hua, C. H., Shukla, H., Ying, X., Nill, S., and Oefke, U. (2008). Proton versus photon radiotherapy for common pediatric brain tumors: comparison of models of dose characteristics and their relationship with cognitive function. Pediatr. Blood Cancer 51, 110-117. doi: 10.1002/pbc.21530

Meyer-Schuman, R., and Antonellis, A. (2017). Emerging mechanisms of aminoacyl-tRNA synthetase mutations in recessive and dominant human disease. Hum. Mol. Genet. 26, R114-R127. doi: 10.1093/hmg/ddx231

Miousse, I. R., Kutanzi, K. R., and Koturbash, I. (2017). Effects of ionizing radiation on DNA methylation: from experimental biology to clinical applications. Int. J. Radiat. Biol. 93, 457-469. doi: 10.1080/09553002.2017.1287454

Parihar, V. K., Pasha, J., Tran, K. K., Craver, B. M., Acharya, M. M., and Limoli, C. L. (2015). Persistent changes in neuronal structure and synaptic plasticity caused by proton irradiation. Brain Struct. Funct. 220, 1161-1171. doi: 10.1007/s00429-014-0709-9

Park, S. G., Schimmel, P., and Kim, S. (2008). Aminoacyl tRNA synthetases and their connections to disease. Proc. Natl. Acad. Sci. U.S.A. 105, 11043-11049. doi: 10.1073/pnas. 0802862105

Raber, J., Allen, A. R., Sharma, S., Allen, B., Rosi, S., Olsen, R. H. J., et al. (2015). Effects of proton and combined proton and 56Fe irradiation on the hippocampus. Radiat. Res. 184, 586-594. doi: 10.1667/RR14103.1
Rabin, B., Buhler, L., Joseph, J., Shukitt-Hale, B., and Jenkins, D. (2002). Effects of exposure to $56 \mathrm{Fe}$ particles or protons on fixed ratio operant responding in rats. J. Radiat. Res. 43(Suppl.), S225-S228. doi: 10.1269/jrr.43.S225

Rabinow, J., Brisman, J., Cole, A., Lee, P., Chapman, P., Loeffler, J., et al. (2006). MRI changes in the rat hippocampus following proton radiosurgery. Stereotact. Funct. Neurosurg. 84, 147-154. doi: 10.1159/000094862

Rudobeck, E., Szucs, A., and Vlkolinsky, R. (2014). Effects of proton radiation on evoked and spontaneous neuronal activity in the hippocampus of APP/PSEN1 transgenice mice. J. Radiat. Res. 55, 202-203. doi: 10.1093/jrr/rrt174

Russell, N. S., Hoving, S., Heeneman, S., Hage, J. J., Woerdeman, L. A., and de Bree, R. (2009). Novel insights into pathological changes in muscular arteries of radiotherapy patients. Radiother. Oncol. 92, 477-483. doi: 10.1016/j.radonc.2009.05.021

Sanchez, M. C., Nelson, G. A., and Green, L. M. (2010). Effects of protons and HZE particles on glutamate transport in astrocytes, neurons, and mixed cultures. Radiat. Res. 174, 669-678. doi: 10.1667/RR2106.1

Sauter, C., Lorber, B., Gaudry, A., Karim, L., Schwenzer, H., Wien, F., et al. (2015). Neurodegenerative disease-associated mutants of a human mitochondrial aminoacyl-tRNA synthetase present individual molecular signatures. Sci. Rep. 5:17332. doi: $10.1038 /$ srep 17332

Shannon, P., Markiel, A., Ozier, O., Baliga, N. S., Wang, J. T., Ramage, D., et al. (2003). Cytoscape: a software environment for integrated models of biomelocular interaction networks. Genome Res. 13, 2498-2504. doi: $10.1101 /$ gr.1239303

Shukitt-Hale, B., Szprengiel, A., Pluhar, J., Rabin, B. M., and Joseph, C. A. (2004). The effects of proton exposure on neurochemistry and behavior. Adv. Space Res. 33, 1334-1339. doi: 10.1016/j.asr.2003.10.038

Simonsen, L. C., Cucinotta, F. A., Atwell, W., and Nealy, J. E. (1993). Temporal analysis of the October 1989 proton flare using computerized anatomical models. Radiat. Res. 133, 1-11. doi: 10.2307/3578250

Sokolova, I. V., Schneider, C. J., Bezaire, M., Soltesz, I., Vlkolinsky, R., and Nelson, G. A. (2015). Proton radiation alters intrinsic and synaptic properties of CA1 pyramidal neurons of the mouse hippocampus. Radiat. Res. 183, 208-218. doi: $10.1667 /$ RR13785.1

Vares, G., Wang, B., Ishii-Ohba, H., Nenoi, M., and Nakajima, T. (2014). Dietinduced obesity modulates epigenetic responses to ionizing radiation in mice. PLoS ONE 9:e106277. doi: 10.1371/journal.pone.0106277

Weintraub, N. L., Jones, W. K., and Manka, D. (2010). Understanding radiation-induced vascular disease. J. Am. Coll. Cardiol. 55, 1237-1239. doi: 10.1016/j.jacc.2009.11.053

Wilson, J. W. (2000). Overview of radiation environments and human exposures. Health Phys. 79, 470-494. doi: 10.1097/00004032-200011000-00005

Xia, J., and Wishart, D. (2010). MetPA: a web-based metabolomics tool for pathway analysis and visualization. Bioinformatics 26, 2342-2344. doi: 10.1093/bioinformatics/btq418

Zhang, Z. Y., Marrachelli, V. G., Yang, W. Y., Trenson, S., Huang, Q. F., Wei, F. F., et al. (2013). Diastolic left ventricular function in relation to circulating metabolic biomarkers in a population study. Eur. J. Prev. Cardiol. 26, 22-32. doi: $10.1177 / 2047487318797395$

Conflict of Interest Statement: The authors declare that the research was conducted in the absence of any commercial or financial relationships that could be construed as a potential conflict of interest.

Copyright $\odot 2019$ Torres, Hall, Bobe, Choi, Impey, Pelz, Lindner, Stevens and Raber. This is an open-access article distributed under the terms of the Creative Commons Attribution License (CC BY). The use, distribution or reproduction in other forums is permitted, provided the original author(s) and the copyright owner(s) are credited and that the original publication in this journal is cited, in accordance with accepted academic practice. No use, distribution or reproduction is permitted which does not comply with these terms. 